Check for updates

Cite this: Mater. Chem. Front., 2019, 3, 1406

Received 31st January 2019,

Accepted 9th April 2019

DOI: 10.1039/c9qm00070d

rsc.li/frontiers-materials

\section{Direct on-surface synthesis of gold-phthalocyanine via cyclization of cyano-groups with gold adatoms $\dagger$}

\author{
Zhongping Wang, ${ }^{a}$ Xiaoqing Liu, ${ }^{a}$ Yan Lu, ${ }^{a}$ Zegao Wang, (D) ${ }^{\text {bc }}$ Christian Bortolini, ${ }^{b}$ \\ Menglin Chen, (DD ${ }^{b}$ Sheng Wei, ${ }^{a}$ Wei Li, ${ }^{a}$ Junfa Zhu, (D) ${ }^{d}$ Huanxin Ju, \\ Federico Rosei, (D) ${ }^{\mathrm{e}}$ Mingdong Dong (D) ${ }^{\mathrm{b}}$ and Li Wang (D) $*^{\mathrm{a}}$
}

We report the synthesis of gold-phthalocyanines on an Au(111) surface via cyclization of dibenzo[fg,op]tetracene-5,6,12,13-tetracarbonitrile molecules. Scanning tunnelling microscopic images unambiguously reveal that gold adatoms act as linkers via metal coordination bonding to form metastable supra-molecular structures, and simultaneously replace metal ions within the final products, metal phthalocyanines.
On-surface single molecule synthesis presents exciting opportunities ${ }^{1-4}$ for producing new functional aromatic architectures, e.g. molecular wires, ${ }^{5}$ two-dimensional porous organic networks, ${ }^{6,7}$ graphene nanoribbons, ${ }^{8,9}$ aromatic polymers and 2D conjugated polymers. ${ }^{10-13}$ Such surface confined polymers ${ }^{14-16}$ have been synthesized using various chemical reactions including Ullmann coupling, ${ }^{17-19}$ Glaser's coupling, ${ }^{20}$ Bergman's cyclization, ${ }^{21}$ cyclodehydrogenation $^{22}$ and cyclo-addition, ${ }^{23}$ where highly active metal atoms play important roles in on-surface synthesis reactions. In addition to surface defects, ${ }^{24}$ foreign atoms ${ }^{25}$ or clusters ${ }^{26}$ deposited on solid surfaces, intrinsic adatoms originating from the substrate may effectively activate or catalyze these reactions, particularly on $\mathrm{Cu}, \mathrm{Pd}$ and Pt surfaces. ${ }^{27}$ Gold is considered as one of the "elements" for classic Ullmann coupling, similar to $\mathrm{Cu}$ and $\mathrm{Ag}$. It is predicted that the chemically inert $\mathrm{Au}$ potentially has enhanced catalytic properties and coordination ability for Ullmann coupling when its size is in the range from one to a few dozens of atoms. Recently, it has been reported that metalorganic coordinated networks can be formed by the interaction of $\mathrm{Au}$ adatoms with organic molecules such as porphyrin or phthalocyanine derivatives, ${ }^{28}$ organo-phosphorus compounds, ${ }^{29}$

\footnotetext{
${ }^{a}$ Department of Physics, Nanchang University, Nanchang 330031, China. E-mail: liwang@ncu.edu.cn

${ }^{b}$ Interdisciplinary Nanoscience Center (iNANO), Sino-Danish Center for Education and Research (SDC), Aarhus University, 8000 Aarhus, Denmark.

E-mail: dong@inano.au.dk

${ }^{c}$ College of Materials Science and Engineering, Sichuan University, Chengdu 610065, China

${ }^{d}$ National Synchrotron Radiation Laboratory, University of Science and Technology of China, Hefei 230029, China

${ }^{e}$ Centre for Energy, Materials and Telecommunications of INRS, Varennes, Quebec J3X 1S2, Canada

$\dagger$ Electronic supplementary information (ESI) available: Experiment and method, author contributions, the rotated and moved STM images, XPS of C and N of HTTN, and STM images of BTTN. See DOI: 10.1039/c9qm00070d
}

cyano-groups $(-\mathrm{C} \equiv \mathrm{N})^{30}$ and molecules containing highly electronegative substituents such as sulphur or chlorine. ${ }^{31}$ Nevertheless, the role of $\mathrm{Au}$ adatoms in chemical reactions, especially as a catalyst for synthesizing novel compounds, is still poorly understood.

Here we present the synthesis of gold-phthalocyanines ( $\mathrm{Au}-\mathrm{Pc}$ ) on an $\mathrm{Au}(111)$ surface via cyclization of dibenzo[ $f g$,op]tetracene5,6,12,13-tetracarbonirile (DTTCN) molecules with gold adatoms. Scanning tunnelling microscopy (STM) images reveal that Au adatoms act as linkers via metal coordination bonding to form metastable supra-molecular structures, and simultaneously replace the metal ions within the final products (i.e. the metal Pc). Our findings shed light on noble metal-based surface synthesis and considerably enrich our understanding of the role of noble metal adatoms in the reaction mechanism.

Fig. 1 displays STM images of the DTTCN molecules on the $\mathrm{Au}(111)$ surface after annealing at various temperatures. Upon annealing at $330 \mathrm{~K}$, DTTCN self-assembles into well-ordered structures where a single DTTCN molecule exhibits a central protrusion with two lateral lobes, as shown in Fig. 1a. By comparing the experimental image and the molecular model of the DTTCN molecule, the central oval protrusion is attributed to an acene-like backbone and two V-shapes with a $60^{\circ}$ angle on the left and the right sides correspond to two adjacent terminal cyano-groups $(-\mathrm{C} \equiv \mathrm{N})$, respectively. After annealing at $450 \mathrm{~K}$ for 5 minutes, the self-assembled structure in Fig. 1a vanishes and is replaced with a new supramolecular structure with a two-fold symmetrical hollow cross-like structure, as shown in Fig. 1b. Each cross-like structure (inset of Fig. 1b) consists of four DTTCN molecules in which the $-\mathrm{C} \equiv \mathrm{N}$ within one side of every DTTCN molecule forms the cyclic ring. This kind of cross-like structure can be rotated and displaced on the surface as a whole during STM manipulations, as shown in Fig. S1 (ESI $\dagger$ ). The stability of this cross-like structure upon STM manipulation confirms that the DTTCN molecules within the new molecular structure are 


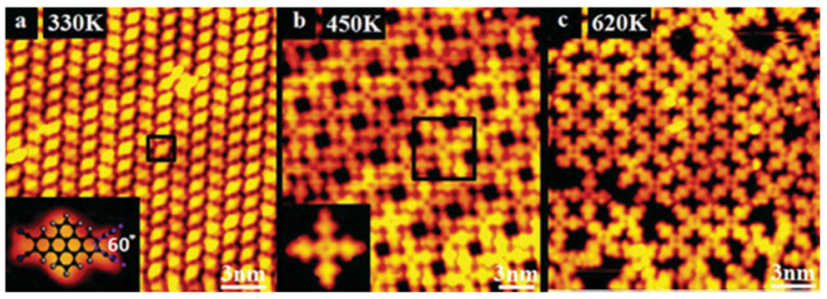

Fig. 1 STM images of DTTCN molecules adsorbed on an Au(111) surface that experience a hierarchical on-surface reaction driven by thermal treatments. (a) The assembly structure; the inset presents the structural model and high-resolution STM (HR-STM) image for a single DTTCN molecule $\left(V_{\mathrm{s}}=-2.8 \mathrm{~V}\right.$ and $\left.I_{\mathrm{s}}=-0.04 \mathrm{nA}\right)$. (b) The cross-like supramolecular network $\left(V_{\mathrm{s}}=-1.80 \mathrm{~V}\right.$ and $\left.I_{\mathrm{s}}=-0.03 \mathrm{nA}\right)$. (c) The mesh-like network $\left(V_{\mathrm{s}}=-1.10 \mathrm{~V}\right.$ and $\left.I_{\mathrm{s}}=-0.03 \mathrm{nA}\right)$.

strongly bonded to each other, indicating that a new compound might be synthesized by combining four DTTCN molecules via cyclization of the $-\mathrm{C} \equiv \mathrm{N}$ on the Au surface.

Abel et $a .^{32,33}$ reported a similar synthesis of metal-Pc, where $\mathrm{Fe}$ or $\mathrm{Mn}$ were introduced as guest metal atoms to the cyclization reaction of the $-\mathrm{C} \equiv \mathrm{N}$. In our study, however, no guest atoms are introduced during annealing, meaning that the reaction occurred as a result of thermal-induced self-assembly and molecular rearrangement. After annealing at $620 \mathrm{~K}$ for $5 \mathrm{~min}$, the DTTCN molecules which are not involved in the reaction at $450 \mathrm{~K}$ to form cross-like structures are activated to take part in the cyclization reactions of cyano-groups; thus, the cross-like structures increase their degree of order by linking each other and aligning along two perpendicular directions, as shown in Fig. 1c.

Based on our experimental observations, the on-surface reaction process of DTTCN molecules driven by thermal treatment can be described as a hierarchical process: (i) every two $-\mathrm{C} \equiv \mathrm{Ns}$ in four DTTCN molecules are activated to take part in the cyclization reaction that leads to the formation of cross-like structures; (ii) the latter act as a precursor to create a mesh-like polymer via the cyclization reaction of the residual cyanogroups within the cross-like molecules.

Fig. 2a identifies a hole at the centre of the cross-like structure surrounded by eight symmetrical spherical protrusions. This feature resembles metal-Pc such as $\mathrm{Cu}-\mathrm{Pc}$ on $\mathrm{HOPG}^{34}$ and $\mathrm{Au}(111),{ }^{35} \mathrm{Zn}-\mathrm{Pc}$ on $\mathrm{Cu}(100) .{ }^{36}$ Abel et al. ${ }^{32,33}$ synthesized Pc-like compounds by using the cyanide of tetracyanobenzene molecules as a precursor and introducing $\mathrm{Mn}$ or $\mathrm{Fe}$ atoms for the metalinduced cyclization reaction. The cross-like structure obtained in this work is very similar to Abel's $\mathrm{Cu}-\mathrm{Pc}^{35}$ and $\mathrm{Fe}-\mathrm{Pc},{ }^{32}$ strongly suggesting that the cross-like compound has a similar chemical structure compared to metal-Pc. Fig. 2b reports the structural model for the Pc-type compound synthesized with $\mathrm{Au}$ as the catalyst by four DTTCN molecules via the cyclization reaction between $-\mathrm{C} \equiv \mathrm{N}$ of DTTCN. Fig. 2c shows the height profiles of the cross-like structure along its perpendicular axes (labelled as $\mathrm{AB}$ and $\mathrm{CD}$ ). The separation between two adjacent protrusions that correspond to the benzene rings located on the side of the DTTCN molecules is measured to be $18 \AA$, which matches the expected value from the structural model in Fig. 2b. Good agreement between the simulated STM image in Fig. 2d and
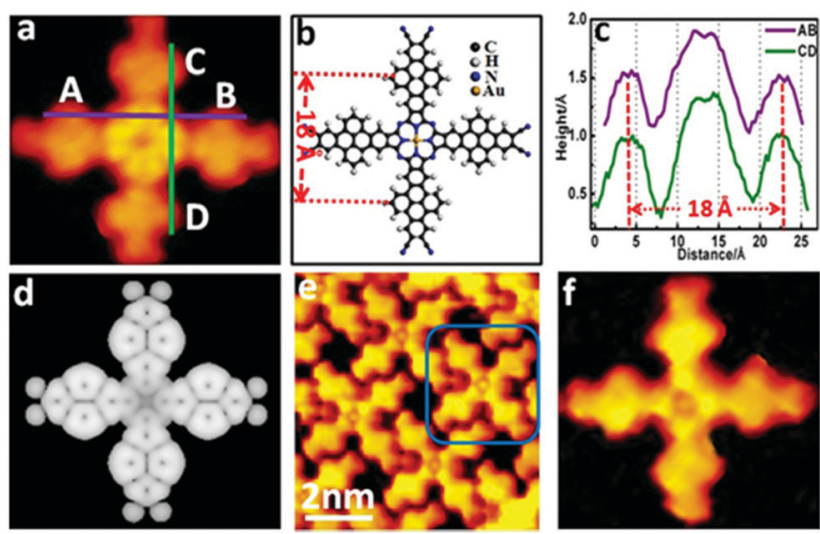

Fig. 2 STM images and models of the cross-like structure at the submolecular level. (a) HR-STM image $\left(V_{\mathrm{s}}=-0.90 \mathrm{~V}\right.$ and $\left.I_{\mathrm{s}}=-0.08 \mathrm{nA}\right)$. (b) Molecular model. (c) Height profiles along the lines indicated in Fig. 2a. (d) Simulated STM image. (e) STM image recorded by functionalizing the STM tip via empirical adsorption of an Au adatom $\left(V_{S}=-0.83 \mathrm{~V}\right.$ and $I_{\mathrm{s}}=-0.10 \mathrm{nA}$ ). (f) Detailed STM image for a single Au-Pc compound.

our experimental results is a strong indication that the crosslike compound possesses a metal-Pc-like chemical structure.

Since none guest metal atoms are introduced onto the surface in our study, it is reasonable to conclude that the coordinated metal atoms in our metal-Pc-like products are actually the Au adatoms, which are incorporated into the Pc rings during the onsite reaction processes. For most metal-Pc molecules (e.g. Fe-Pc ${ }^{32}$ and $\left.\mathrm{Cu}-\mathrm{Pc}^{34}\right)$, their coordinated metal atoms are not visible in both the experimental and simulated STM images although the coordinated Co atom appears as a bright dot in CoPc metal. ${ }^{37}$ In convex metal-Pc (e.g. $\mathrm{Pb}-\mathrm{Pc})$, the $\mathrm{Pb}$ atom appears as a bright dot. $^{38}$ To test whether the coordinated Au atom is present or not in such compound, the STM image has been obtained at the single molecule level by functionalizing the STM tip via empirical adsorption of a Au adatom from the Au(111) surface, as shown in Fig. 2e. The visibility of the coordinated Au atom in the centre of the cross-like structure provides further insights into our compound being a Au-Pc-like structure, as shown in Fig. 2f. With the assistance of $\mathrm{Au}$ adatoms, the $-\mathrm{C} \equiv \mathrm{N}$ of the DTTCN molecules participates in the on-surface cyclization reaction to synthesize $\mathrm{Au}-\mathrm{PC}$-like molecules, while the DTTCN core (that possesses thermal-stability) allows the main skeleton of the DTTCN molecule to be retained, even upon annealing at $620 \mathrm{~K}$. The surface cyclization of $-\mathrm{C} \equiv \mathrm{N}$ with $\mathrm{Au}$ adatoms is also confirmed by core-level measurements of the different elements (C and N), as shown in Fig. S2 (ESI $\dagger$ ). This $\mathrm{Au}-\mathrm{PC}$-like molecule can be named with Au-(dibenzo[fg,op]tetrancene) porphyrazine which is abbreviated as Au-DTTporphyrazine. Due to the similar molecular configuration, ${ }^{39}$ we propose that the form of gold in the Au-DTT-porphyrazine is $3+$. The experiment results show only $\mathrm{Au}-\mathrm{DTT}$-porphyrazine oligomer packing monolayer rather than a covalent Au-DTTporphyrazine network. In this case one side phthalonitrile of one DTTCN is activated to form an Au-DTT-porphyrazine oligomer but at the same time the other side phthalonitrile will be passivated. 


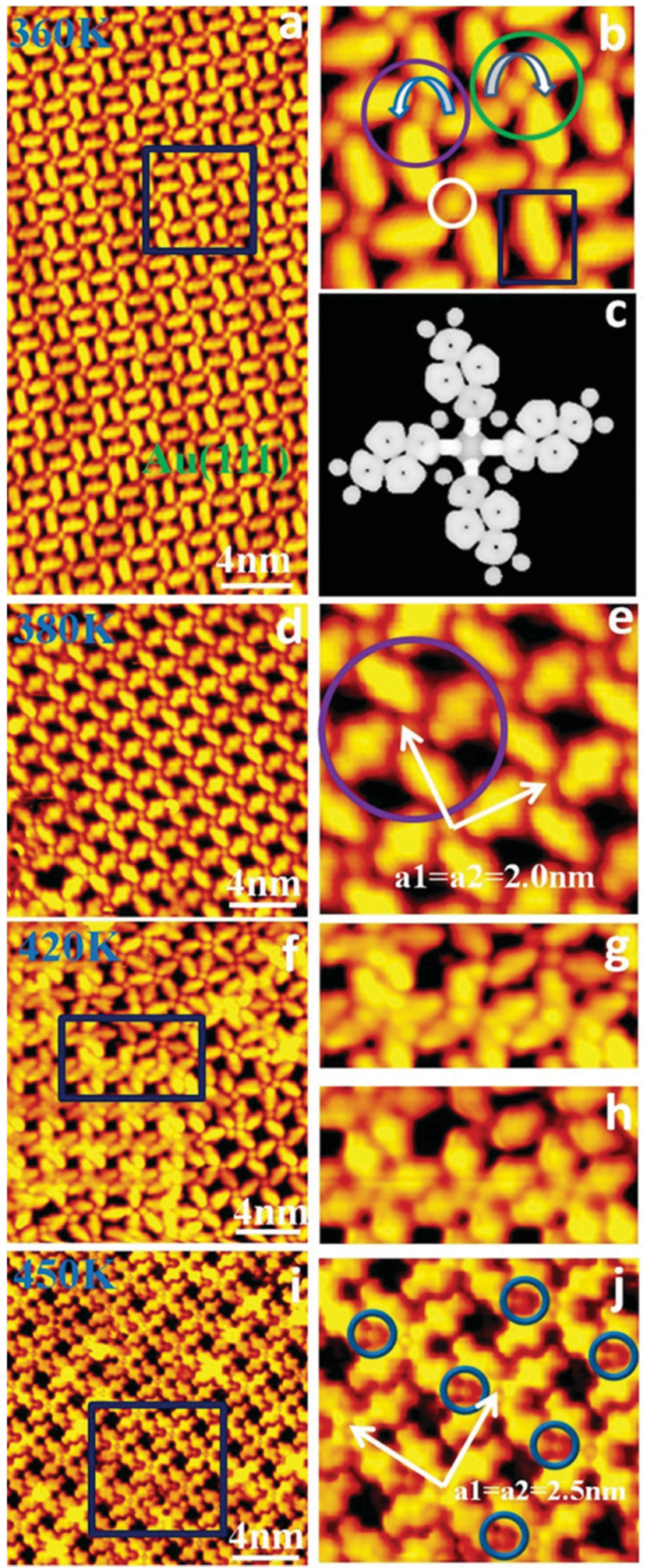

Fig. 3 Observation of a cross-like structure on-surface synthesis stepby-step. (a) Windmill-like configuration upon annealing at $360 \mathrm{~K}$ $\left(V_{\mathrm{s}}=-0.65 \mathrm{~V}\right.$ and $\left.I_{\mathrm{s}}=-0.04 \mathrm{nA}\right)$. (b) Zoomed-in image and (c) simulated image of the windmill-like configuration with a left-hand structure. (d) Large-area and (e) HR-STM image of the left-handed structures by annealing at $380 \mathrm{~K}\left(V_{\mathrm{s}}=-1.16 \mathrm{~V}\right.$ and $\left.I_{\mathrm{s}}=-0.05 \mathrm{nA}\right)$. (f) The coexistence of the windmill-like arrangement and the Au-Pc compound after annealing at $420 \mathrm{~K}\left(V_{\mathrm{s}}=-0.88 \mathrm{~V}\right.$ and $\left.I_{\mathrm{s}}=-0.05 \mathrm{nA}\right)$. (g) HR-STM image for the area marked by a square in (f). (h) STM image of the same area in (g) acquired with an unfunctionalized tip $\left(V_{\mathrm{s}}=-1.30 \mathrm{~V}\right.$ and $\left.I_{\mathrm{s}}=-0.03 \mathrm{nA}\right)$. (i) Assembled structure and (j) HR-STM image of Au-DTT-porphyrazine upon annealing at $450 \mathrm{~K}\left(V_{\mathrm{s}}=-0.85 \mathrm{~V}\right.$ and $\left.I_{\mathrm{s}}=-0.1 \mathrm{nA}\right)$. Au adatoms are labelled with a blue circle. (STM images in (a), (b), (d)-(g), (i), and (j) acquired by functionalizing the STM tip via empirical adsorption of an Au ad-atom on the $A u(111)$ surface).

Fig. 3 shows the step-by-step reaction from DTTCN molecules to Au-DTT-porphyrazine formation during thermal treatments ranging from $360 \mathrm{~K}$ to $450 \mathrm{~K}$. As shown in Fig. 3a, even if the reconstruction of the $\mathrm{Au}(111)$ surface is present, annealing at $360 \mathrm{~K}$ drives the DTTCN molecules to self-assemble into windmill-like structures. Four DTTCN molecules gathering around a dot, assumed as a windmill-like configuration with either a right-hand (green circle) or a left-hand (purple circle) structure, form long-range ordered $2 \mathrm{D}$ domains by sharing a single DTTCN molecule among adjacent windmill-like structures, as shown in Fig. 3b. The circular dots located at the centre of the windmill-like structures are considered as trapped $\mathrm{Au}$ adatoms originated from the $\mathrm{Au}(111)$ surface rather than being extracted from the crystals. This conclusion is drawn on the basis that no guest atoms were introduced to the $\mathrm{Au}(111)$ surface before or after the adsorption of the DTTCN molecules, and we did not observe increase of the lattice imperfections on the surface. These windmill-like structures are composed of $\mathrm{N}$ atoms of $-\mathrm{C} \equiv \mathrm{N}$ coordinated to the $\mathrm{Au}$ adatom and the two adjacent $-\mathrm{C} \equiv \mathrm{N}$ with an angle of $60^{\circ}$ in a molecular chemical structure, leading to the formation of ordered left/right-handed metal coordinated structures. A simulated STM image for the metal-organic coordinated windmill-like structure with left-hand is shown in Fig. 3c. The features observed in this image are similar to those found in the experimental STM topography. This mixed structure gradually converts to the left-handed or right-handed metal coordinate structure as a result of an increase of annealing treatment temperature to $380 \mathrm{~K}$. An ordered windmill-like arrangement with left-handed structures is shown in Fig. 3d, with a close-up view presented in Fig. 3e. Two white arrows highlight the square unit cell containing two molecules and one $\mathrm{Au}$ atom; the vectors were measured to be $a 1=a 2=2.0 \mathrm{~nm}$ with an angle of $90^{\circ}$. As shown, if the annealing temperature is further increased to $420 \mathrm{~K}$ (for 5 minutes), two types of co-existing building blocks are obtained. It is therefore possible to observe the transition between the well-ordered arrangement (Fig. 3d) and the new self-assembly structure highlighted (with a blue rectangle) in Fig. 3f. Fig. $3 \mathrm{~g}$ shows the STM image corresponding to the blue rectangle in Fig. 3f. If these structures are imaged by using a normal tip (Fig. 3h), the coordinated Au atoms in the windmill-like structures are not visible. Therefore, new Au-DTT-porphyrazine molecules are obtained from a reaction between DTTCN molecules and $\mathrm{Au}$ ad-atoms provided by the $\mathrm{Au}(111)$ surface. Further increasing the annealing treatment to $450 \mathrm{~K}$, as shown in Fig. 3i, the formation of a homogeneous pattern of coordinated $\mathrm{Au}-\mathrm{DTT}$-porphyrazine structures on the $\mathrm{Au}(111)$ surface is observed. In addition, the brighter dots labelled by blue circles in Fig. 3j indicate that the gold adatoms assist the DTTCN 
molecules' reaction to form Au-DTT-porphyrazine and simultaneously promote the coordination of the Au-DTT-porphyrazine products to form a metal-organic morphology with a periodic arrangement. To further understand the role of the gold adatom in the reaction, the tert-butyl groups are chosen to substitute the $\mathrm{H}$ atoms in positions 2 and 9 in the DTTCN molecule to form 2,9-diter-butyldibenzo[fg,op]tetracene-5,6,12,13-tetracarbonirile molecules (BTTN). Fig. S3 (ESI $\dagger$ ) unambiguously confirms that the cyano-groups within the BTTN are not able to coordinate with the gold adatoms, because these functional groups are not in the same plane of the gold adatoms. These observations confirm that the gold ad-atom plays an important role in surface cyclization reactions to form noble metal Pc.

\section{Conclusions}

In summary, we report the confined synthesis of gold-Pc molecules on an $\mathrm{Au}(111)$ substrate via a reaction between DTTCN molecules and gold adatoms. STM observations directly reveal that the gold ad-atoms act as linkers via metal coordination bonding to form metastable supra-molecular structures, and simultaneously replace metal ions within the final products, metal-Pc. This finding sheds new light on understanding the role of noble metal ad-atoms in on-surface reactions. In addition, the hierarchical synthesis of a $\pi$-conjugated system embedding gold ad-atoms is a key step forward towards chemical synthesis using 2D confinement of precursor molecules on metal surfaces, and the bottom-up growth of functional architectures for future electronic and optoelectronic devices.

\section{Conflicts of interest}

There are no conflicts to declare.

\section{Acknowledgements}

This work was financially supported by the Natural Science Foundation of China (Grant No. 61474059, 11727902 and 61864007) and the Natural Science Foundation of Jiangxi Province (20181BAB201012). L. W. acknowledges Jiangxi Provincial Innovation Talents of Science and Technology (20165BCB18003). M. D. acknowledges grants from the Danish National Research Foundation, Aarhus Universitets Forskningsfond (AUFF NOVAproject), Karen Elise Jensens Fond, Villum Foundation, Lundbeck Foundation, and EU H2020 RISE 2016 (MNR4SCell 734174 project). F. R. is grateful to the Canada Research Chairs program for partial salary support. Z. W. acknowledges grants from Fundamental Research Funds for the Central Universities, China (YJ201893).

\section{Notes and references}

1 C. Santato and F. Rosei, Nat. Chem., 2010, 2, 344.

2 M. Ebrahimi and F. Rosei, Nature, 2017, 542, 423.
3 M. El Garah, J. M. MacLeod and F. Rosei, Surf. Sci., 2013, $613,6$.

4 X. Wang, J. K. Weber, L. Liu, M. Dong, R. Zhou and J. Li, Nanoscale, 2015, 7, 15341.

5 Z. Wang, H. H. Wu, Q. Li, F. Besenbacher, X. C. Zeng and M. Dong, Nanoscale, 2018, 10, 18178.

6 J. Eichhorn, D. Nieckarz, O. Ochs, D. Samanta, M. Schmittel, P. J. Szabelski and M. Lackinger, ACS Nano, 2014, 8, 7880.

7 Y. Li, L. Liu, R. Subramani, Y. Pan, B. Liu, Y. Yang, C. Wang, W. Mamdouh, F. Besenbacher and M. Dong, Chem. Commun., 2011, 47, 9155.

8 P. Han, K. Akagi, C. F. Federici, R. Shimizu, H. Oguchi, S. Shiraki, P. S. Wsiss, N. Asao and T. Hitosugi, ACS Nano, 2015, 9, 12035.

9 Z. Wang, H. Zhao, Y. Lu, F. Xiang, X. Leng, X. Liu, X. Song, M. Dong and L. Wang, Chem. Commun., 2016, 52, 8420.

10 Z. Wang, Q. Li, H. Xu, C. Dahl-Petersen, Q. Yang, D. Cheng, D. Cao, F. Besenbacher, J. V. Lauritsen, S. Helveg and M. Dong, Nano Energy, 2018, 49, 634.

11 L. Lafferentz, V. Eberhardt, C. Dri, C. Africh, G. Comelli, F. Esch, S. Hecht and L. Grill, Nat. Chem., 2012, 4, 215.

12 D. Peyrot and F. Silly, ACS Nano, 2016, 10, 5490.

13 Z. Wang, Q. Li, Y. Chen, B. Cui, Y. Li, F. Besenbacher and M. Dong, NPG Asia Mater., 2018, 10, 703.

14 G. Franc and A. Gourdon, Phys. Chem. Chem. Phys., 2011, 13, 14283.

15 Q. Fan, C. Wang, L. Liu, Y. Han, J. Zhao, J. Zhu, J. Kuttner, G. Hilt and J. M. Gottfried, J. Phys. Chem. C, 2014, 118, 13018.

16 M. Bieri, M. T. Nguyen, O. Gröning, J. Cai, M. Treier, K. AïtMansour, P. Ruffieux, C. A. Pignedoli, D. Passerone and M. Kastler, et al., J. Am. Chem. Soc., 2010, 132, 16669.

17 M. Di Giovannantonio, M. Tomellini, J. Lipton-Duffin, G. Galeotti, M. Ebrahimi, A. Cossaro, A. Verdini, N. Kharche, V. Meunier and G. Vaseur, et al., J. Am. Chem. Soc., 2016, 138, 16696.

18 E. A. Lewis, C. J. Murphy, M. L. Liriano and E. C. H. Sykes, Chem. Commun., 2014, 50, 1006.

19 J. Eichhorn, T. Strunskus, A. Rastgoo-Lahrood, D. Samanta, M. Schmittel and M. Lackinger, Chem. Commun., 2014, 50, 7680 .

20 Y. Q. Zhang, N. Kepčija, M. Kleinschrodt, K. Diller and S. Fischer, Nat. Commun., 2012, 3, 1286.

21 Q. Sun, C. Zhang, Z. Li, H. Kong, Q. Tan, A. Hu and W. Xu, J. Am. Chem. Soc., 2013, 135, 8448.

22 G. Otero, G. Biddau, C. Sánchez-Sánchez, R. Caillard, M. F. López, C. Rogero, F. J. Palomares, N. Cabello, M. A. Basanta and J. Ortega, et al., Nature, 2008, 454, 865.

23 O. Diaz Arado, H. Mönig, H. Wagner, J. H. Franke, G. Langewisch, P. A. Held, A. Studer and H. Fuchs, ACS Nano, 2013, 7, 8509.

24 M. Kong, Y. Li, X. Chen, T. Tian, P. Fang, F. Zheng and X. Zhao, J. Am. Chem. Soc., 2011, 133, 16414.

25 W. Thijssen, D. Marjenburgh, R. Bremmer and J. M. Van Ruitenbeek, J. Phys. Rev. Lett., 2006, 96, 026806.

26 D. Y. Murzin, J. Catal., 2010, 276, 85. 
27 S. T. Marshall and J. W. Medlin, Surf. Sci. Rep., 2011, 66, 173. 28 J. Mielke, F. Hanke, M. V. Peters, S. Hecht, M. Persson and L. Grill, J. Am. Chem. Soc., 2015, 137, 1844.

29 A. D. Jewell, E. C. H. Sykes and G. Kyriakou, ACS Nano, 2012, 6, 3545 .

30 M. N. Faraggi, N. Jiang, N. Gonzalez-Lakunza, A. Langner, S. Stepanow, K. Kern and A. Arnau, J. Phys. Chem. C, 2012, 116, 24558.

31 W. Xu, M. Dong, H. Gersen, E. Rauls, S. Vazquez-Campos, M. Crego-Calama, D. N. Reinhoudt, E. Laegsgaard, I. Stensgaard and T. R. Linderoth, Small, 2008, 4, 1620.

32 S. Kezilebieke, A. Amokrane, M. Abel and J. P. Bucher, J. Phys. Chem. Lett., 2014, 5, 3175.

33 M. Koudia and M. Abel, Chem. Commun., 2014, 50, 8565.
34 Y. L. Huang, H. Li, J. Ma, H. Huang, W. Chen and A. T. Wee, Langmuir, 2009, 26, 3329.

35 E. Nardi, L. Chen, S. Clair, M. Koudia, L. Giovanelli, X. Feng, K. Müllen and M. Abel, J. Phys. Chem. C, 2014, 118, 27549.

36 F. Chen, X. Chen, L. Liu, X. Song, S. Liu, J. Liu, H. Ouyang, Y. Cai, X. Liu and H. Pan, Appl. Phys. Lett., 2012, 100, 081602.

37 D. E. Barlow, L. Scudiero and K. W. Hipps, Langmuir, 2004, 20, 4413.

38 A. Sperl, J. Kroger and R. Berndt, J. Phys. Chem. A, 2011, 115, 6973.

39 S. Mullegger, W. Schofberger, M. Rashidi, T. Lengauer, F. Klappenberger, K. Diller, K. Kara, J. V. Barth, E. Rauls, W. G. Schmidt and R. Koch, ACS Nano, 2011, 5, 6480. 